\title{
The 3D solar minimum with differential emission measure tomography
}

\author{
Alberto M. Vásquez ${ }^{1,2}$, Richard A. Frazin ${ }^{3}$, Zhenguang Huang ${ }^{3}$, \\ Ward B. Manchester IV $^{3}$ and Paul Shearer ${ }^{3}$ \\ ${ }^{1}$ Instituto de Astronomía y Física del Espacio (CONICET-UBA), \\ CC 67 - Suc 28, (C1428ZAA) Ciudad de Buenos Aires, Argentina \\ email: albert@iafe.uba.ar \\ ${ }^{2}$ Facultad de Cs. Exactas y Naturales, Universidad de Buenos Aires, Argentina \\ ${ }^{3}$ Deptartment of Atmospheric, Oceanic and Space Sciences, \\ University of Michigan, Ann Arbor, MI 48109, USA
}

\begin{abstract}
Differential emission measure tomography (DEMT) makes use of extreme ultraviolet (EUV) image series to deliver two products: a) the three-dimensional (3D) reconstruction of the coronal emissivity in the instrumental bands, and b) the 3D distribution of the local differential emission measure (LDEM). The LDEM allows, in turn, construction of 3D maps of the electron density and temperature distribution. DEMT is being currently applied to the space-based EUV imagers, allowing reconstruction of the inner corona in the height range 1.00 to $1.25 \mathrm{R}_{\odot}$. In this work we applied DEMT to different Carrington Rotations corresponding to the last two solar Cycle minima. To reconstruct the 2008 minimum we used data taken by the Extreme UltraViolet Imager (EUVI), on board the Solar TErrestrial RElations Observatory (STEREO) spacecraft, and to reconstruct the 1996 minimum we used data taken by the Extreme ultraviolet Imaging Telescope (EIT), on board the Solar and Heliospheric Observatory (SOHO). We show here comparative results, discussing the observed $3 \mathrm{D}$ density and temperature distributions in the context of global potential magnetic field extrapolations. We also compare the DEMT results with other observational and modeling efforts of the same periods.
\end{abstract}

Keywords. Sun: corona, Sun: fundamental parameters, Sun: UV radiation, methods: data analysis

\section{Introduction}

The increasing capability of today's computers allows for increasingly complex global MHD models of the solar corona. This calls for the development of global 3D constraints for the fundamental coronal parameters, such as the electron density and temperature. Such developments are of interest in themselves, in order to observationally study the $3 \mathrm{D}$ structure of coronal features.

The DEMT technique, described in detail in Frazin et al. (2009) (FVK09 hereafter), allows reconstruction of the 3D distribution of the coronal DEM. In a first stage, time series of EUV images are used to reconstruct de 3D distribution of the filter band emissivity (FBE) via solar rotational tomography (SRT), for each band of the telescope independently. In a second stage, the values of the FBEs reconstructed in each tomographic grid cell are used to invert for a parametrized local-DEM (LDEM) pertaining only to the plasma contained in that cell.

In previous works we used DEMT to study two rotations of the solar Cycle 23 minimum phase, namely the Carrington Rotation (CR-)2068 period (Vásquez et al. (2011), V11 hereafter), which was the object of coordinated study efforts (known as the Whole Heliosphere Interval, WHI), and CR-2077 (Vásquez et al. (2010), V10 hereafter), which 
corresponds to the absolute minimum of solar Cycle 23. Both works used STEREO/EUVI data. In the present work we revisit those two periods, updating the ionization equilibrium calculations used to compute the temperature responses of each instrumental band, and including comprehensive 3D comparative results. In addition, we show results for the solar minimum of the previous solar Cycle 22, using SOHO/EIT data of CR-1914.

Fig. 1 shows the normalized temperature responses of the coronal bands of both the STEREO/EUVI-B and the SOHO/EIT instruments, computed using CHIANTI v6.0.1, assuming the Feldman et al. (1992) abundance set, and the Bryans et al. (2009) ionization equilibrium calculations.

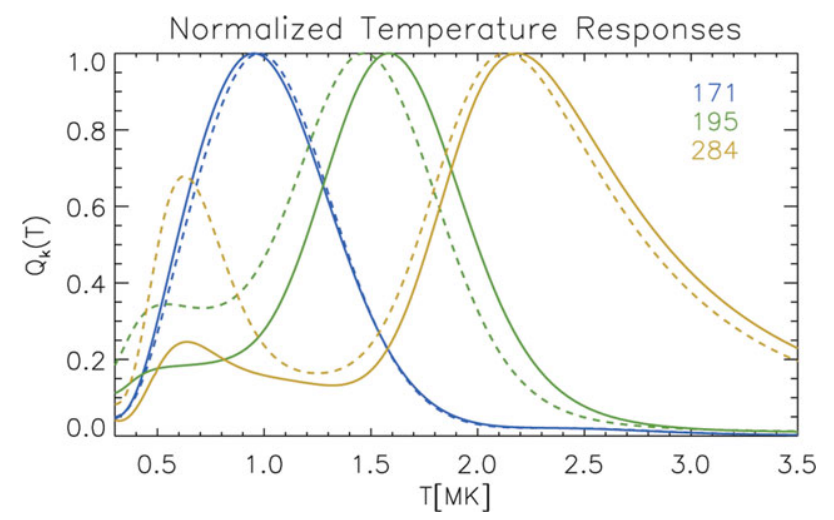

Figure 1. Temperature responses of STEREO/EUVI (solid) and SOHO/EIT (dashed).

\section{Reconstructing the 3D FBE and Determining the LDEM}

In this work, the inner corona $\left(1.0-1.25 \mathrm{R}_{\odot}\right)$ is discretized on a $25 \times 90 \times 180$ (radial $\times$ latitudinal $\times$ longitudinal) spherical tomographic grid. As an example of the results of the first stage of DEMT, Fig. 2 shows the FBE maps for CR-2077 and CR-1914 at a selected height of the tomographic grid, and for the three bands of EUVI and EIT. The saturated regions correspond to zero density artifacts (ZDAs) in the reconstructions, which are locations of reconstructed zero emissivity, and are the result of coronal dynamics acting during the data acquisition while SRT assumes a static coronal structure.

The overploted thick-solid curves indicate the open/closed boundary of PFSS models, based on MDI synoptic magnetograms for each period, while the solid-thin white (black) curves are contour levels of positive (negative) magnetic strength. The PFSS models are computed using the finite-difference iterative solver FDIPS by Tóth et al. (2011), on a $150 \times 180 \times 360$ spherical grid, covering 1.0 to $2.5 \mathrm{R}_{\odot}$. There is an overall good agreement between the location of the brighter-FBE structures and the PFSSM-closed regions, especially clear in the 195 and $284 \AA$ bands. This is consistent with the closed plasma having enhanced densities and temperatures, implying larger emissivities.

To find the temperature distribution of the plasma within each tomographic cell, we assume a parametrization for the LDEM and use the FBE values to derive the parameters by minimizing the quadratic deviations between the tomographic and the predicted FBE in all bands. The instruments used in the present work (EUVI and EIT) have three bands each one, and we parametrize the LDEM as a single-normal distribution in each tomographic cell. With the new Atmospheric Imaging Assembly (AIA) instrument, on board the Solar Dynamics Observatory (SDO) spacecraft, the number of bands is increased, and other parametrizations can be explored (see Nuevo et al., 2012, in this volume). 

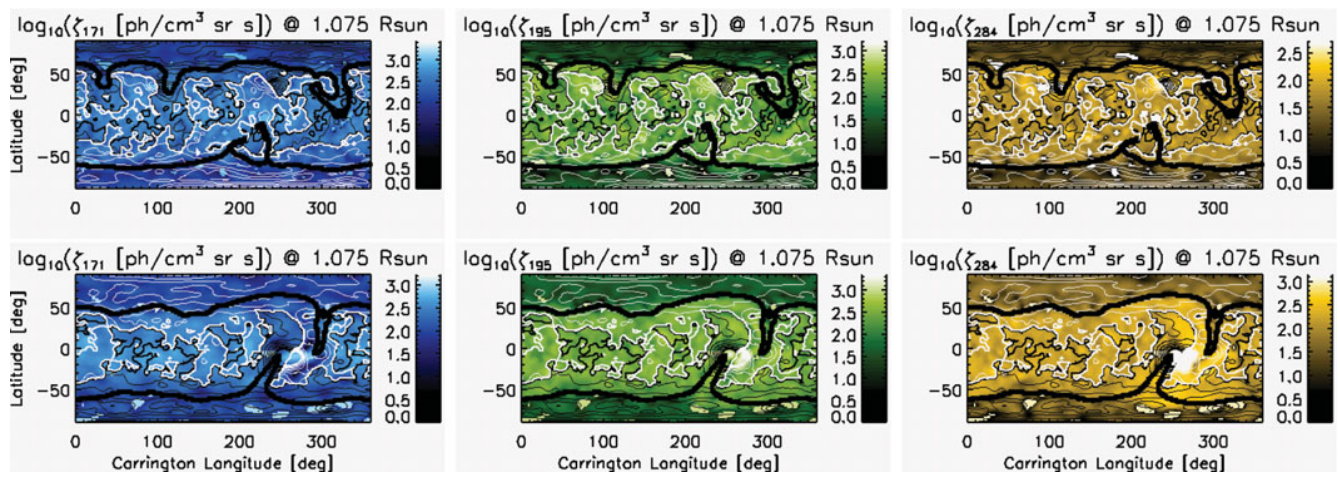

Figure 2. Examples of tomographically reconstructed emissivities at $1.075 \mathrm{R}_{\odot}$ for the CR-2077 (top panels) and CR-1914 (bottom panels) periods, in the 171 (right), 195 (middle), and $284 \AA$ (left) coronal bands. The CR-2077 reconstructions are based on STEREO/EUVI-B data, while the CR-1914 reconstructions are based on SOHO/EIT data. The saturated regions correspond to ZDAs in the reconstructions (see text). The thick-solid curves indicate the open/closed boundary of the PFSS models for each period, while the solid-thin white (black) curves are contour levels of positive (negative) magnetic strength. All figures in this paper were designed to be seen in color in the online version, where their visibility is highly improved.

Using only three bands, a single-normal function is able to accurately predict the three tomographic FBEs in most regions. This is quantitatively shown in detail in Fig. 3 where, for three heights of the tomographic grid, we display the mean relative quadratic deviation ( $\chi^{2}$, see Eq. (1.4) in Nuevo et al., 2012, in this volume for details) between the tomographic and predicted FBEs, as synthesized from the LDEM in each tomographic cell for the three periods we analyzed. We thresholded the lower values at $\chi^{2}=10^{-3}$, or an average agreement between the synthetic and tomographic FBEs better than $\sim 3 \%$, which are the dark-green regions in the maps. It is readily seen that in most of the coronal volume the agreement is as good as, or superior, to this value.

The black regions correspond to ZDAs, where no LDEM is computed. The white regions indicate tomographic grid cells where $\chi^{2}>5 \times 10^{-2}$, or an average disagreement of about $20 \%$ between the tomographic and synthetic FBEs. We consider those cells to have nonsatisfactory-fits (NSF) of their LDEM, i.e. regions where the assumed parametrization is not able to reasonably reproduce the tomographic FBEs. The NSFs usually occur around ZDAs, as well as in near-polar fainter regions. These regions are characterized by unusual relative values of the FBE in the three bands, making the single-normal function unable to reproduce them. A clear example of this is readily seen in the case of the CR-1914 period, where NSFs extend over a large range of longitudes in the northern hemisphere near-polar region. This is due to the data being contaminated by a filter leak in the $284 \AA$ band near the north pole. The abnormally enhanced $284 \AA$ signal makes a single-normal function unable to reproduce the three bands FBE with accuracy.

\section{Global 3D Maps of Solar Minimum DEMT Results}

Once the LDEM is found at each tomographic grid cell, its zeroth through second moments (Eqs. (1.1)-(1.3) in Nuevo et al., 2012, in this volume) give the squared electron density $N_{e}^{2}$, mean electron temperature $T_{m}$, and squared electron temperature spread $W_{T}^{2}$, respectively. In Fig. 4 we show the results corresponding to CR-2077 at three different heights of the tomographic grid, for all latitudes and longitudes. In Figs. 5 and 6 we show the corresponding results for CR-2068 and CR-1914, respectively. 

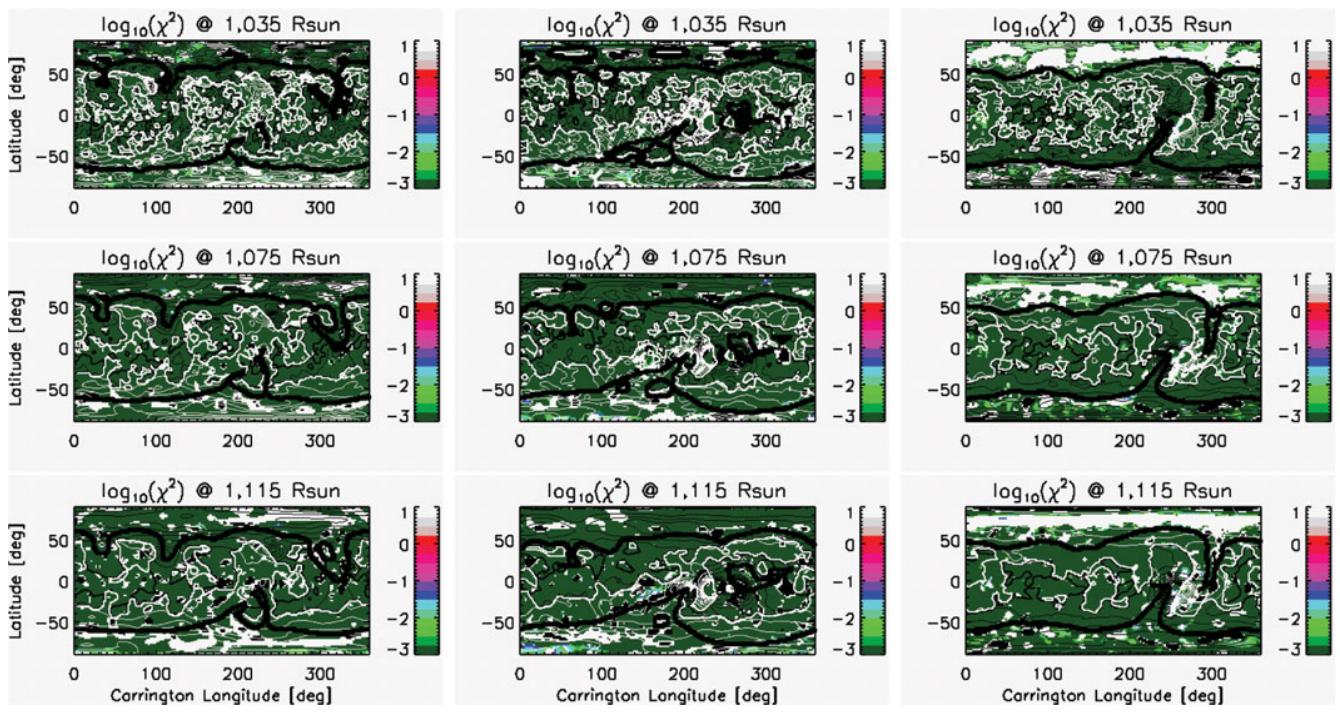

Figure 3. Mean cuadratic deviation $\left(\chi^{2}\right)$ between the tomographic and predicted FBE at three heights of the tomographic grid, for CR-2077 (right panels), CR-2068 (middle panels), and CR-1914 (left panels).

Several characteristics are common to all three tomographic reconstructions and PFSS models. Firstly, there is an overall good agreement in the location of the PFSS model open/closed (O/C) boundary and the location of the DEMT electron density and temperature transition between enhanced values within the closed regions and lower values in the open regions. Exceptions to this are usually related to low latitude incursions of open regions, especially when they are narrow and more $\mathrm{S}-\mathrm{N}$ oriented. We believe this is the result of smearing of the results due to the fact that our current implementation of SRT does not consider coronal differential rotation. There is also the issue of coronal dynamics, but these effects should be minimized at the minimum of the cycle. Secondly, the largest temperature regions are systematically located within closed regions, along and around polarity inversion lines. Across the $\mathrm{O} / \mathrm{C}$ boundary the change in temperature is quite sharp, which is shown in detail in Figs. 11 and 12.

To globally quantify the differences between open and closed regions, Figs. 7 through 9 show histograms of $N_{e}, T_{m}$, and $W_{T}$ at $1.075 \mathrm{R}_{\odot}$, separately for the open and closed regions. We find a similar 2:1 ratio for the closed-to-open electron density contrast, with the electron density median value being about 10\% larger for the CR-1914 period, compared to the CR-2077 and CR-2068 periods. We find the median $T_{m}$ to be $30 \%$ larger in the 1996 closed region, compared to the 2008 periods. Differences in temperature could be partly due to the different instruments used. In inverting simulated DEM synthetic data (not included in this work), we found no systematic differences in the EUVI and EIT results. On the other hand, stray light issues are different for both instruments and have not yet been taken into account. Still, we have applied DEMT to the CR-2077 dataset using a preliminary blind deconvolution of the EUVI images (Shearer \& Frazin, 2011) and found that the median temperature results are not greatly affected in the streamer region. All of these elements support the idea that the difference found in the temperature of the closed regions is a physical one, with the 2008 period being about $30 \%$ cooler than the 1996 one. In all the periods we found a median $T_{m}<1 \mathrm{MK}$ in open regions, which is consistent with other studies revealing sub-MK electron temperatures at high latitudes. 

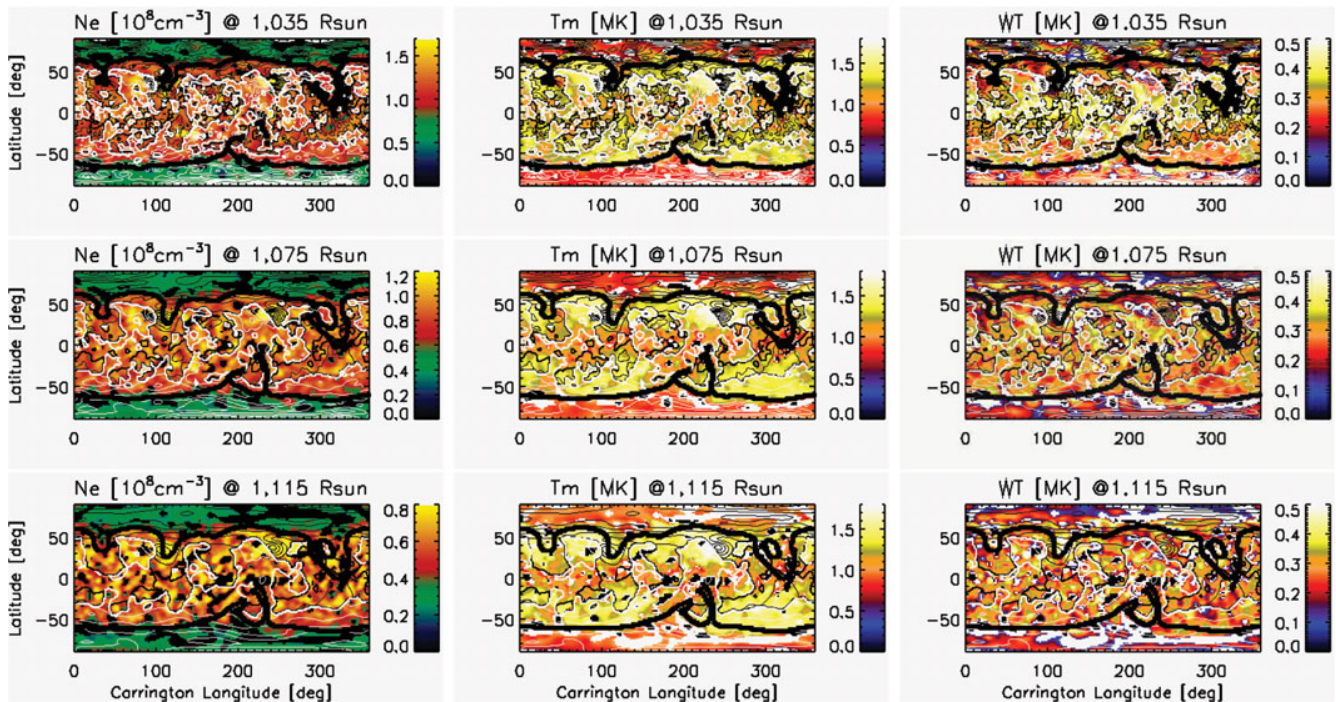

Figure 4. Reconstructed maps of $N_{e}$ (left panels), $T_{m}$ (middle panels), and $W_{T}$ (right panels) for CR-2077, at heights 1.035 (top), 1.075 (middle), and $1.115 \mathrm{R}_{\odot}$ (bottom), based on STEREO/EUVI data. In the temperature maps the black regions correspond to ZDAs, while the white regions to NSFs (see text), while both regions are shown as black in the density maps. The thick-solid curves indicate the open/closed boundary of the PFSS models for the period, while the solid-thin white (black) curves are contour levels of positive (negative) magnetic strength.
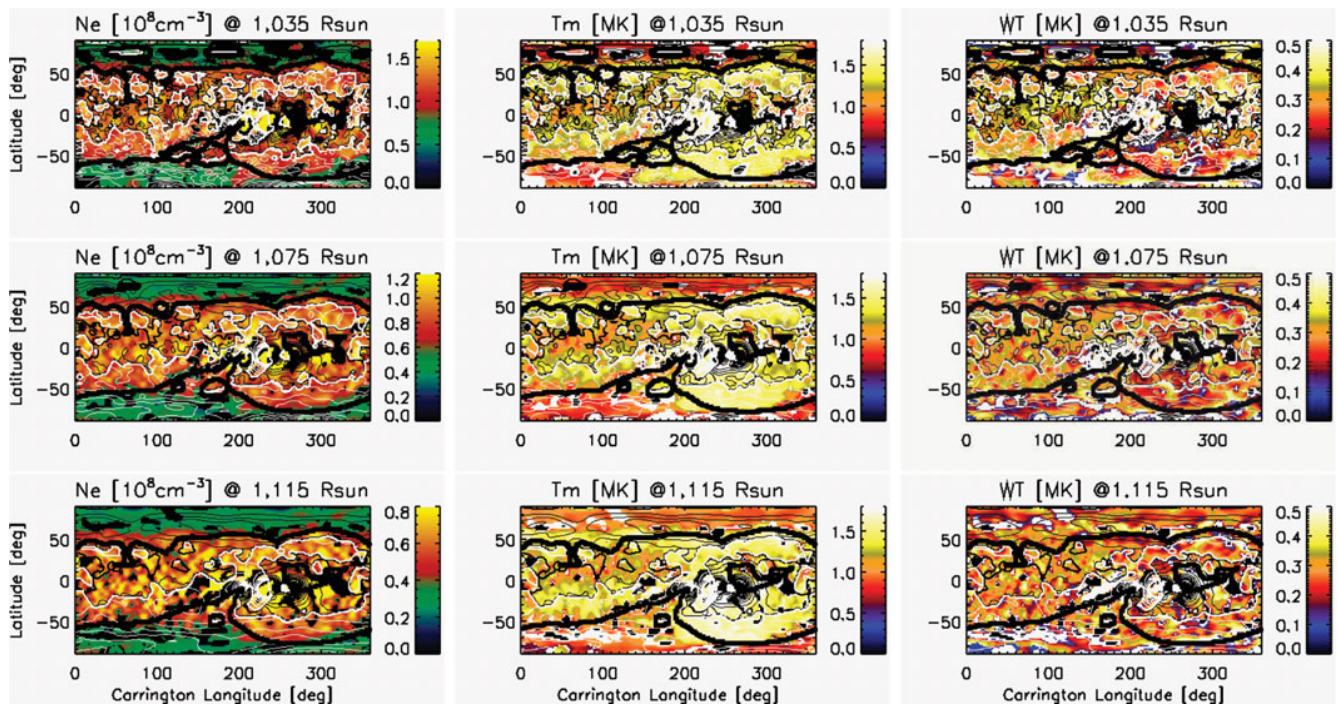

Figure 5. Same as Fig. 4 but for the CR-2068 period, based on STEREO/EUVI data.

Using the LDEM $N_{e}$ and $T_{m}$ values to estimate the gas total thermal pressure as $p_{\text {th }} \sim 2 k_{B} N_{e} T_{m}$ and the magnetic strength of the PFSS model to compute the magnetic pressure $p_{B} \equiv B^{2} / 8 \pi$, Fig. 10 shows plasma $\beta \equiv p_{\text {th }} / p_{B}$ maps at $1.075 \mathrm{R}_{\odot}$. The CR2077 and CR-1914 periods, both corresponding to a deep solar minimum, show more $\mathrm{N} / \mathrm{S}$ symmetry than CR-2068. This is due to this last period belonging to the declining phase of Cycle 23 but still showing multipolar components from the AR complex, which clearly affected the overall structure as the polar fields were particularly weak during 

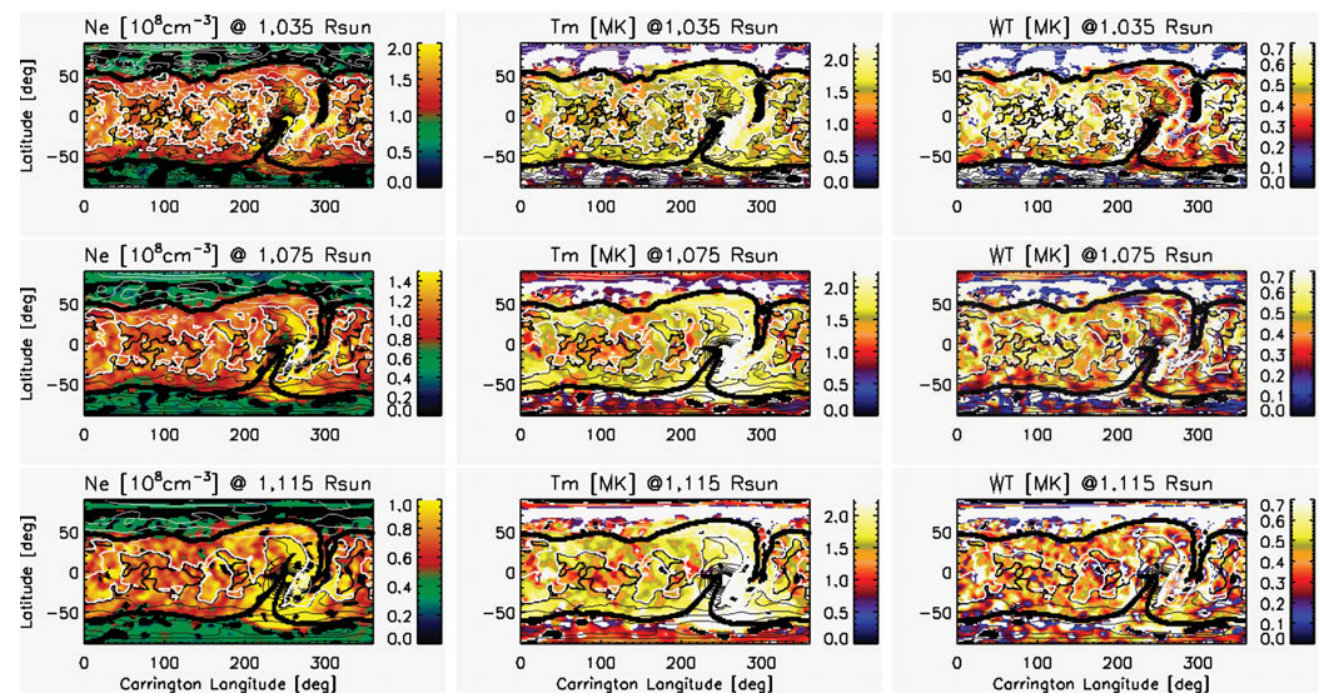

Figure 6. Same as Fig. 4 but for the CR-1914 period, based on SOHO/EIT data.
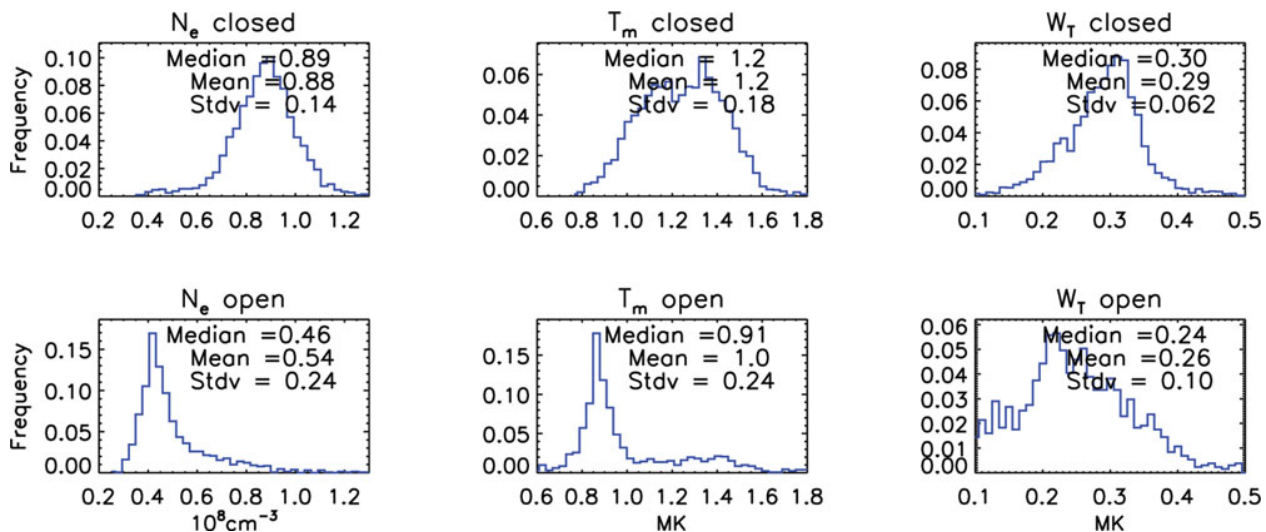

Figure 7. Histograms of $N_{e}, T_{m}$, and $W_{T}$ at $1.075 \mathrm{R}_{\odot}$, for the open and closed regions of CR-2077.

this period. We find $\beta>1$ in streamer cores, similar to the Li et al. (1998) study of the July 1996 streamer based on SXT and UVCS data, and a PFSS extrapolation, which estimated $\beta \sim 5$ at $1.15 \mathrm{R}_{\odot}$.

\section{DEMT Results as a Function of Latitude and Height}

To analyze the large scale corona latitudinal dependence of the DEMT results during both minima we select, for the periods CR-2077 and CR-1914, the ranges of more quiet longitudes and average the results in longitude. For CR-1914 we select the range of longitudes $\left[340^{\circ}, 150^{\circ}\right]$. For CR-2077 we select the ranges $\left[0^{\circ}, 20^{\circ}\right],\left[50^{\circ}, 100^{\circ}\right],\left[130^{\circ}, 170^{\circ}\right]$, $\left[250^{\circ}, 280^{\circ}\right]$, and $\left[340^{\circ}, 360^{\circ}\right]$, which correspond to the more quiet and simply organized corona. In both periods we are then averaging over a longitude range spanning about $180^{\circ}$. The results for CR-2077 and CR-1914 are displayed in the top three panels of Figs. 11 and 12 , respectively. 

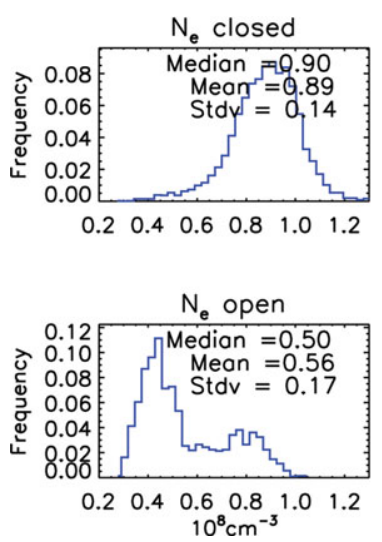

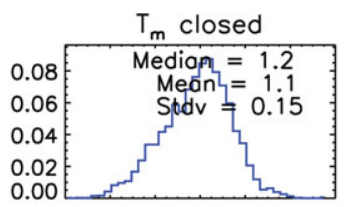

$\begin{array}{llllllllll}0.6 & 0.8 & 1.0 & 1.2 & 1.4 & 1.6 & 1.8\end{array}$

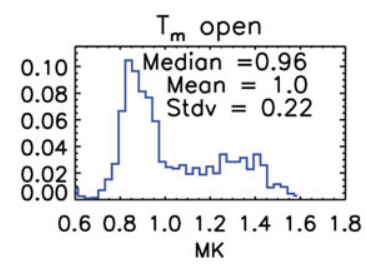

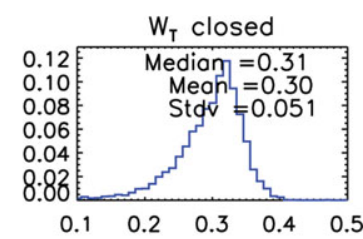

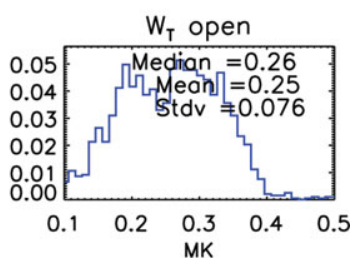

Figure 8. Same as Fig. 7 but for CR-2068.
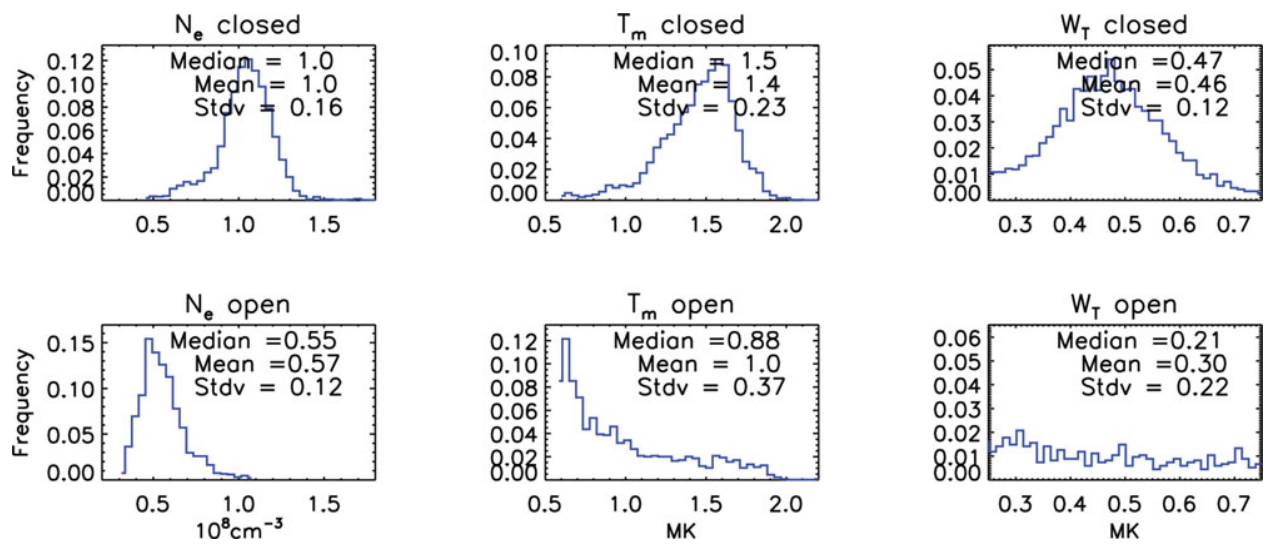

Figure 9. Same as Fig. 7 but for CR-1914.
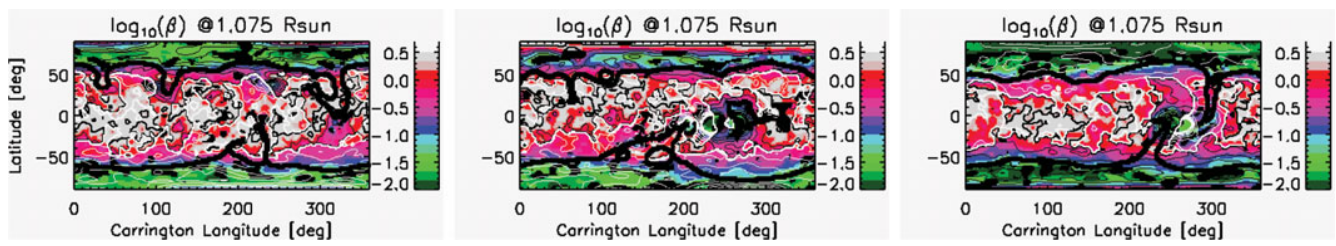

Figure 10. Plasma- $\beta$ maps for CRs 2077 (right), 2068 (middle), and 1914 (left), at $1.075 \mathrm{R}_{\odot}$.

In both periods the electron density $N_{e}$ is always more enhanced in the center of the closed regions, corresponding to the streamer belt core region, with a contrast of order 2:1 compared to the subpolar regions. About $70 \%$ of the transition of density between the streamer and subpolar regions occurs across a $\sim 20^{\circ}$ layer around the $\mathrm{O} / \mathrm{C}$ boundary.

In both periods the mean electron temperature $T_{m}$ is larger at mid-latitudes, within the closed regions, showing always a quite sharp transition to lower values across the $\mathrm{O} / \mathrm{C}$ boundary. In both minima, within the closed region of large scale streamers covering both hemispheres, as for example from longitudes $0^{\circ}$ to $150^{\circ}$ for CR-2077 and the same range of longitudes for CR-1914, the temperature is lower at its central latitudes than near the $\mathrm{O} / \mathrm{C}$ boundary. This latitudinal variation of temperature is similar to that found by Wilhelm et al. (2002). They performed a 1998 streamer study based on SUMER data and found larger $T_{e}$ at mid latitudes and lower values in the streamer central latitudes (1.4 

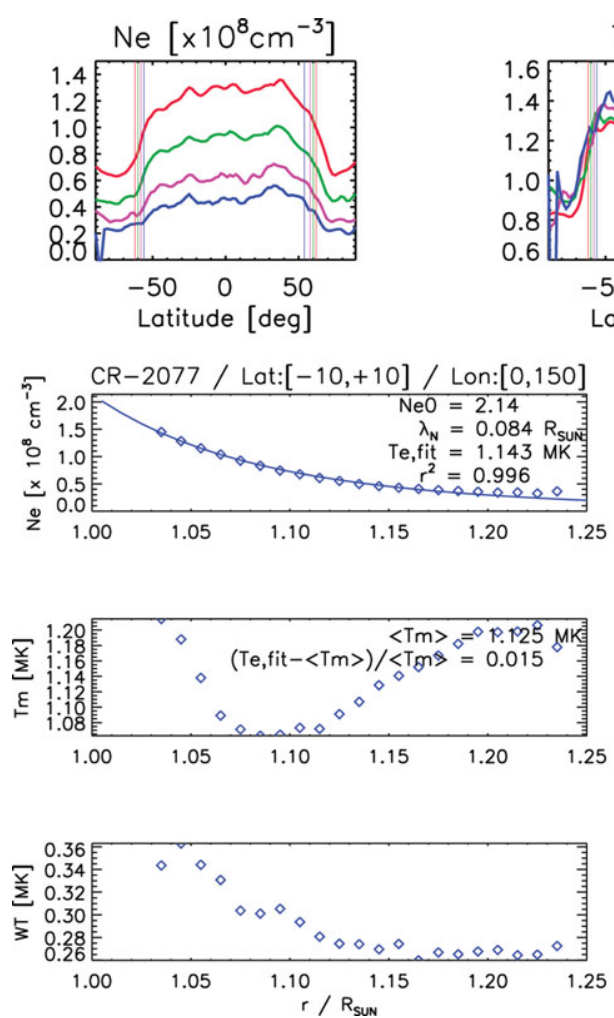
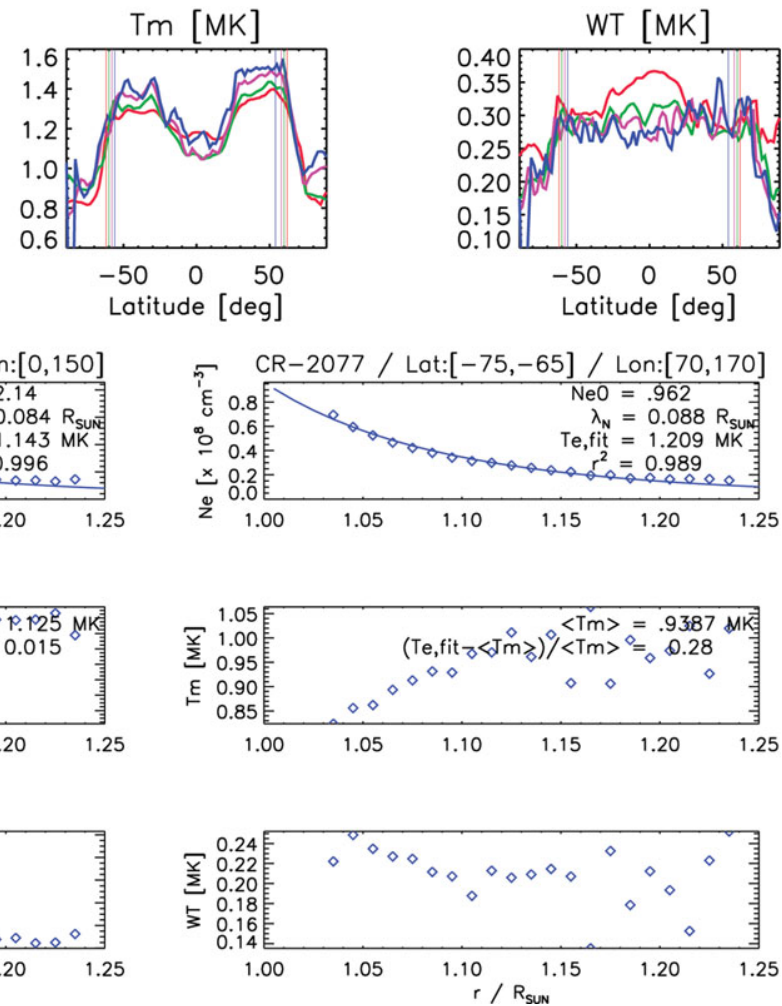

Figure 11. Averaged DEMT results for CR-2077. Top three panels: longitudinal average of the latitudinal variation for the quiet longitudes (see text) at four heights: 1.035 (red), 1.075 (green), 1.115 (magenta), and $1.155 \mathrm{R}_{\odot}$ (blue). For each height, using the same color code, the vertical lines indicate the average latitude of the $\mathrm{O} / \mathrm{C}$ boundary in both hemispheres. Bottom Panels: average dependence with height for the streamer (left) and subpolar (right) regions. The colours correspond to the on line version of the paper.

and 1.0 MK, respectively). In CR-2068, the temperatures are also found to be enhanced in both streamer and pseudo-streamer regions, seen between longitudes $250^{\circ}$ and $350^{\circ}$, in the southern and northern mid latitudes, respectively, surrounding the equatorial coronal hole seen in Fig. 5 (see also Figs. 4 and 8 in Gibson et al., 2011).

The temperature spread $W_{T}$ also presents a quite sharp transition to lower values right outside the $\mathrm{O} / \mathrm{C}$ boundary, but its behavior within the streamer is a bit different than for $T_{m}$. At lower heights $\left(1.035\right.$ and $\left.1.075 \mathrm{R}_{\odot}\right)$ there is an indication of an anti-correlation between both quantities, with $W_{T}$ achieving larger values where $T_{m}$ shows a minimum in the central part of the streamer core (see top right panel in Figs. 11 and 12).

Figs. 11 and 12 also show the average dependence with height of LDEM results in both the streamer central latitudes and in the subpolar regions, averaged over different ranges of longitudes and latitudes, as indicated in each plot. In each analyzed region we applied an isothermal hydrostatic (HS) fit to the LDEM $N_{e}(r)$ data and found the electron temperature $T_{e}$,fit from the fit scale height, assuming $T_{e}=T_{\mathrm{H}}$. Taking the LDEM $\left\langle T_{m}\right\rangle$ as a measure of the true $T_{e}$, one can use the expression of the HS fit height scale temperature to solve for the proton temperature (Vásquez et al. 2011), to find $\frac{T_{\mathrm{H}}}{\left\langle T_{m}\right\rangle} \approx 1+2\left(\frac{T_{e}, \text { fit }-\left\langle T_{m}\right\rangle}{\left\langle T_{m}\right\rangle}\right)$. 

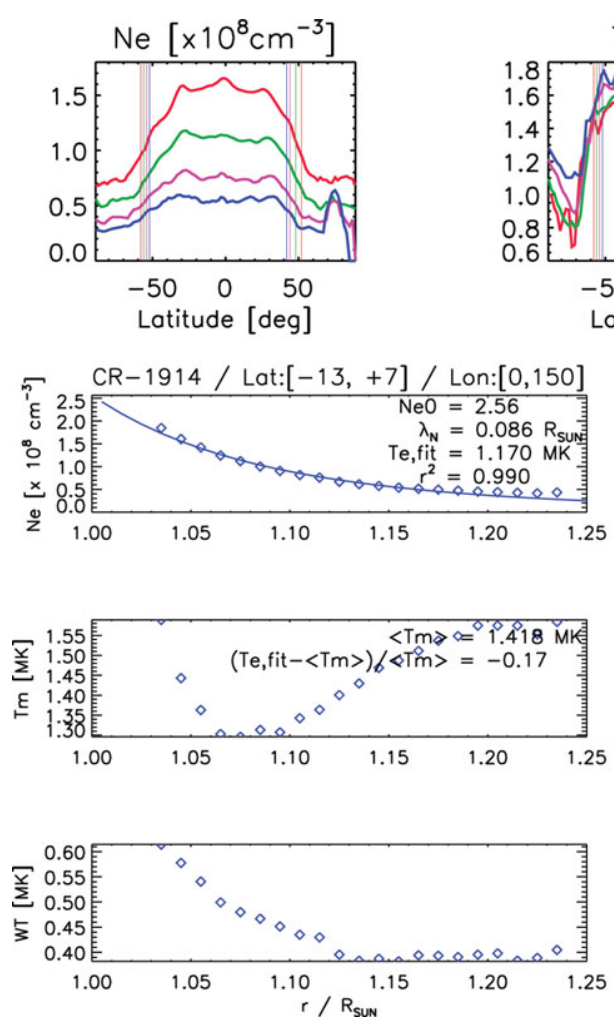
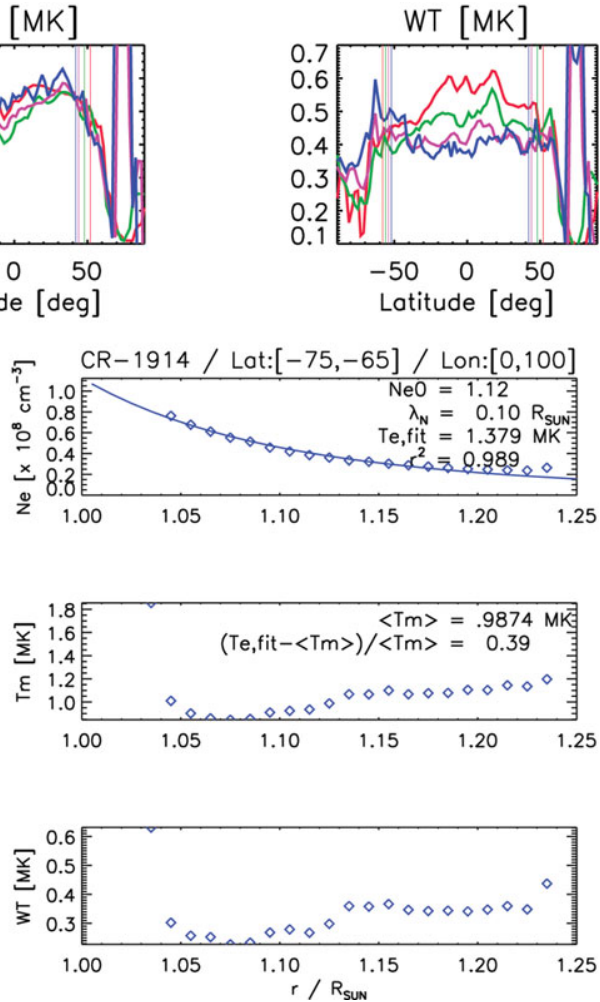

Figure 12. CR-1914. Top three panels: latitudinal variation of the results at four heights. Bottom Panels: average dependence with height for the streamer (left) and subpolar (right) regions.

The variation with height of the LDEM $N_{e}$ in the CR-2077 streamer is consistent with the isothermal HS fit, as the correlation coefficient of the fit $r^{2}$ is high and also $T_{e, \text { fit }} \sim\left\langle T_{m}\right\rangle$. We find the same for the CR-2068 streamer (not shown here due to space limitations). The CR-1914 streamer results present a different behavior, with also a high correlation coefficient but with $T_{e, \text { fit }}$ being about $17 \%$ lower compared to $\left\langle T_{m}\right\rangle$. This may indicate a HS situation with excess electron temperature compared to protons. In the open subpolar regions all three periods show similar characteristics: a high value of the correlation coefficient (a bit lower than in the streamers though), and $\left.T_{e, \text { fit }}\right\rangle\left\langle T_{m}\right\rangle$, with relative differences between 30 and $40 \%$. This is consistent with either proton excess temperatures or the presence of pressure mechanisms other than thermal that are linear with the electron density (see Vásquez et al. 2011 for a detailed CR-2068 analysis).

\section{Concluding Remarks}

We have performed DEMT analysis of three solar minimum rotations, two corresponding to the minimum phase at the end of solar Cycle 23, the third one corresponding to the minimum at the end of solar Cycle 22. In the case of the solar Cycle 23 periods, we are revisiting previous analysis (see Vásquez et al. 2010, 2011), updating the ionization equilibrium calculations assumed in the emission model to that of Bryans et al. (2009), and also providing here more comprehensive comparative figures of the $3 \mathrm{D}$ distribution of the results, along with detailed information on the degree of success of the parametrized LDEM to accurately predict the tomographically inverted emissivities. We analyzed all 
three periods in the context of PFSS magnetic extrapolations corresponding to each period, which were also improved compared to previous analysis. Some conclusions that arise from this analysis are as follows,

(a) The streamer:subpolar electron density ratio is of order 2:1 in all three periods. About $\sim 70 \%$ of transition occurs over a $\sim 20^{\circ}$ latitude width layer around the $\mathrm{O} / \mathrm{C}$ boundary. Note that the DEMT electron density is proportional to $1 / \sqrt{[\mathrm{Fe}]}$, where $[\mathrm{Fe}]$ is the iron abundance, which has been assumed uniform in this work.

(b) In streamers, the LDEM $N_{e}(r)$ and $T_{m}(r)$ are quite consistent with isothermal hydrostatic equilibrium over the range of heights 1.0 to $1.2 \mathrm{R}_{\odot}$.

(c) The largest temperatures are always found within streamers and pseudo-streamers, along and around polarity inversion lines. These regions are located at mid latitudes, near the $\mathrm{O} / \mathrm{C}$ boundary, while the central latitudes of the streamer show relatively lower temperatures, still above $1 \mathrm{MK}$.

(d) Open subpolar regions are characterized by sub-MK electron temperatures. There is in general a quite sharp latitudinal gradient of temperature at the $\mathrm{O} / \mathrm{C}$ boundary.

(e) In open regions surrounding streamers, we also find HS fits to present low residuals, but characterized by $T_{e, \text { fit }}>T_{m}$. This is consistent with either pressure mechanisms other than thermal (but linear in density) being present and/or $T_{\mathrm{H}}>T_{e}$ (up to $\sim 50 \%$ larger, see also Vásquez et al., 2011)

(f) The CR-1914 minimum streamer is 30\% hotter than for CR-2077. Based on the PFSS models, the CR-2077 streamer had an 8\% larger basal area than both the CR-2068 and the CR-1914 streamers.

Future improvements of DEMT include the use of SDO/AIA data for more extensive temperature constraints on the LDEM inversion (a currently undergoing effort, see Nuevo et al., 2012, in this volume) and validation of the DEMT results with Markov chain Monte Carlo (MCMC) simulations. We are also carrying on a study of DEMT inversion of simulated DEM with different available EUV imagers (EIT, EUVI, AIA), in order to learn how to interpret differences of DEMT results obtained with data from different instruments.

Regarding future improvements of the tomographic inversion of emissivities, the consideration of coronal differential rotation has the potential to allow for better resolution of N-S oriented elongated structures. The effects of dynamics in static tomographic reconstructions can be mitigated by using time-dependent approaches, as the Kalman-filtering method (Frazin et al., 2005; Butala et al., 2010). Local DEM analysis can then be applied to produce a time-dependent version of the DEMT technique. Stray light contamination due to the point-spread-function (PSF) affects all EUV imagers. Efforts to perform PSF blind-deconvolution of both STEREO/EUVI and SDO/AIA imagers are being developed by Shearer \& Frazin (2011). Future inclusion of this procedures in the tomographic data pre-processing pipeline will greatly improve DEMT analysis.

DEMT major sources of uncertainty include the tomographic inversion regularization level, uncertainties in the assumed atomic data to compute the temperature response of the EUV imagers, and the coronal dynamics effects. All these factors combined lead to uncertainties of up to about $30 \%$ in the LDEM moments. A comprehensive study of DEMT uncertainties will be the subject of a future publication.

\section{Acknowledgements}

A.M.V. acknowledges the IAU for their support to attend the IAU Symposium 286 at the City of Mendoza, Argentina. 


\section{References}

Bryans, P., Landi, E., \& Savin, W. 2009, ApJ, 691, 1540-1559

Butala, M. D., Hewett, R. J., Frazin, R. A., \& Kamalabadi, F. 2010, Solar Phys., 262, 495-509

Feldman, U., Mandelbaum, P., Seely, J. L., Doschek, G. A., \& Gursky, H. 1992, ApJ, 81, 387-408

Frazin, R. A., Kamalabadi, F., \& Weber, M. A. 2005, ApJ, 628, 1070-1080

Frazin, R. A., Vásquez, A. M., \& Kamalabadi, F. 2009, ApJ, 701, 547-560

Gibson, S. E., de Toma, G., Emery, B., Riley, P., Zhao, L.,Elsworth, Y., Leamon, R. J., Lei, J., McIntosh, S., Mewaldt, R. A., Thompson, B. J., \& Webb, D. 2011, Sol. Phys., 274, 5-27

Li, J., Raymond, J. C., Acton, L. W., Kohl, J. L., Romoli, M., Noci, G., \& Naletto, G. 1998, ApJ, 506, 431-438

Nuevo, F. A., Vásquez, A. M., Frazin, R. A., Huang, Z., \& Manchester IV, W. B. 2012, this volume

Shearer, P. \& Frazin, R. A. 2011, Phys. Rev. Lett., submitted

Tóth, G., van der Holst, B., \& Huang, Z. 2011, ApJ, 732, 102-108

Vásquez, A. M., Frazin, R. A., \& Manchester IV, W. B. 2010, ApJ, 715, 1352-1365

Vásquez, A. M., Huang, Z., Manchester IV, W. B., \& Frazin, R. A. 2011, Sol. Phys., 274, 259-284

Wilhelm, K., Inhester, B., \& Newmark, J. S. 2002, Astron. Astrophys., 382, 328-341

\section{Discussion}

LINKER: Have you tried to take your emission measure and $\mathrm{T}$ values to compare with XRT?

VÁSQUEZ: No, we have not yet done so, but that is something worth exploring. Thank you.

GiBson: Were your bimodal population seen in the density and temperature histograms of the open regions related to the bimodal winds described by Giuliana (de Toma) in her talk?

VÁSQUEZ: Actually, slide 12 displays the frequency histograms for the $N_{e}, T_{m}$, and $W_{T}$ values at $1.075 \mathrm{R}_{\odot}$, for the magnetically open and closed regions separately, covering the whole range of longitudes. To establish a correlation with the solar wind speed, it would be necessary to study the DEMT results along field lines in the MHD model and compare with the velocities of the model along the lines. We will also look into this. 